\title{
Embrella embolic deflection device for cerebral protection during transcatheter aortic valve replacement
}

\author{
Mariam Samim, MD, ${ }^{\mathrm{a}}$ Pierfrancesco Agostoni, MD, PhD, ${ }^{a}$ Jeroen Hendrikse, MD, PhD, ${ }^{\mathrm{b}}$ \\ Ricardo P. J. Budde, MD, PhD, ${ }^{b}$ Freek Nijhoff, MD, ${ }^{a}$ Jolanda Kluin, MD, PhD, ${ }^{c}$ Faiz Ramjankhan, MD, \\ Pieter A. Doevendans, MD, PhD, ${ }^{a}$ and Pieter R. Stella, MD, $\mathrm{PhD}^{\mathrm{a}}$
}

\begin{abstract}
Aims: To compare the extent of cerebral ischemic injury after transcatheter aortic valve replacement (TAVR) with the use of an Embrella Embolic Deflector System versus unprotected TAVR.

Methods: Fifteen patients with severe symptomatic aortic stenosis underwent TAVR with use of the Embrella Embolic Deflector System for cerebral protection. Cerebral diffusion-weighted magnetic resonance imaging (DWI) was performed in all patients at day 4 after the procedure and images were retrospectively compared to 37 patients who had previously undergone TAVR without a protection device (TAVR-only group).
\end{abstract}

Results: Successful placement of the Embrella device was achieved in all patients. DWI revealed an increase in the number of ischemic lesions in the Embrella group compared with the TAVR-only group (9.0 vs 5.0, $P=.044$ ). The use of the Embrella device was however associated with a significant reduction in singlelesion volume: $9.7 \mu \mathrm{L}[5.8,18.4]$ versus $17.8 \mu \mathrm{L}[9.5,38.7](P<.001)$. Moreover, total infarct volumes of more than $1000 \mu \mathrm{L}$ were only seen in the TAVR-only group. More lesions occurred in the right side of the brain in the Embrella group, whereas in the TAVR-only group lesions were distributed equally between left and right. One patient in the TAVR-only group suffered from a transient ischemic attack. Postoperative evaluation was clinically uneventful in the Embrella group.

Conclusions: The use of the Embrella device during TAVR increased the number of cerebral ischemic lesions on postprocedural brain imaging. This increase in number was however accompanied by a significant reduction in single-lesion volume and the absence of large total infarct volumes. (J Thorac Cardiovasc Surg 2015;149:799-805)

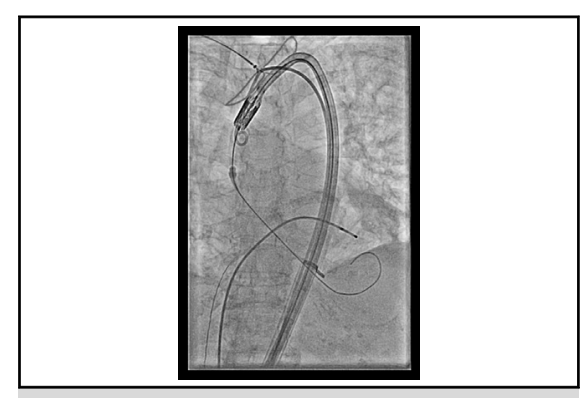

Embrella Embolic Deflector System for brain protection during transcatheter aortic valve replacement.

\section{Central Message}

We evaluated the benefit of the Embrella Embolic Deflector System for cerebral protection during TAVR. The use of this device during TAVR increased the number of cerebral ischemic lesions on postprocedural brain imaging, although it significantly reduced lesion volume as compared to procedures performed without cerebral protection.

\section{Author Perspective}

TAVR is associated with a high incidence of new cerebral ischemic lesions. The use of an EPD may reduce the frequency of TAVR-related embolic events. The curren study shows that the use of Embrella device for brain protection during TAVR is associated with a higher number of postprocedural cerebral DWI lesions. This increase in number is, however, accompanied by a significant reduction in lesion volume, the absence of large lesions, and a trend toward lower total infarct volume as compared to procedures without EPD usage. This reduction in infarct volumes may lower the risk of future neurocognitive impairment and needs further investigation.

See Editorial Commentary pages 806-7.
$\mathcal{B}$ Supplemental material is available online.

Transcatheter aortic valve replacement (TAVR) is an accepted treatment option for inoperable or high-risk patients with severe aortic stenosis. Although good procedural

\footnotetext{
From the Departments of Cardiology, ${ }^{\mathrm{a}}$ Radiology, ${ }^{\mathrm{b}}$ and Thoracic Surgery, ${ }^{\mathrm{c}}$ University Medical Centre, Utrecht, The Netherlands.

Received for publication March 6, 2014; revisions received April 25, 2014; accepted for publication May 9, 2014; available ahead of print Nov 15, 2014.

Address for reprints: Pieter R. Stella, MD, PhD, Department of Cardiology, University Medical Center Utrecht, Heidelberglaan 100, Room E.04.210, 3584 CX Utrecht, The Netherlands (E-mail: p.stella@umcutrecht.nl). $0022-5223 / \$ 36.00$

Copyright (c) 2015 by The American Association for Thoracic Surgery http://dx.doi.org/10.1016/j.jtcvs.2014.05.097
}

success and favorable clinical outcomes have been reported, ${ }^{1,2}$ issues remain regarding the relatively high complication rate. One of the most important drawbacks of TAVR is the risk of intraprocedural cerebral embolization causing brain injury. In high-risk surgical candidates, TAVR is associated with an approximately 2-fold increased incidence of stroke or transient ischemic attack (TIA) $(5.5 \%$ vs $2.4 \%, P=.04)$ at 30 days as compared with surgical aortic valve replacement. ${ }^{3}$ Moreover, new foci of restricted cerebral perfusion on diffusion-weighted magnetic resonance imaging (DWI) are reported in 58\% to $91 \%$ of patients undergoing TAVR. ${ }^{4-8}$ The clinical impact of these new, usually asymptomatic DWI lesions is still uncertain. However, epidemiologic studies have reported an association between asymptomatic cerebral 


$$
\begin{aligned}
& \text { Abbreviations and Acronyms } \\
& \mathrm{CABG}=\text { coronary artery bypass grafting } \\
& \text { DWI = diffusion-weighted magnetic resonance } \\
& \text { imaging } \\
& \text { EPD }=\text { embolic protection device } \\
& \text { FLAIR }=\text { fluid-attenuated inversion recovery } \\
& \mathrm{IQR}=\text { interquartile range } \\
& \text { MRI = magnetic resonance imaging } \\
& \text { MSCT }=\text { multislice computed tomography } \\
& \text { PCI = percutaneous coronary intervention } \\
& \mathrm{SD}=\text { standard deviation } \\
& \text { TAVR }=\text { transcatheter aortic valve replacement } \\
& \text { TE }=\text { echo time } \\
& \text { TIA }=\text { transient ischemic attack } \\
& \mathrm{TR}=\text { repetition time }
\end{aligned}
$$

infarctions and frailty, decline in cognitive and physical functions, early development of dementia, and an increased risk of subsequent stroke. ${ }^{9,10}$ In order to reduce the risk of these potentially devastating adverse events and in the light of progressive movement of TAVR toward younger lower-risk patients, significant research has focused on identifying the risk factors of TAVR-related cerebral embolization and methods for reducing the risk of cerebral ischemic injury. Embolic protection devices (EPDs) have already demonstrated their benefit in carotid artery interventions. ${ }^{11}$ This would suggest that the application of a similar concept for brain protection could minimize the embolic burden during TAVR as well.

We report our experience with the Embrella Embolic Deflector System (Edwards Lifesciences Ltd, Irvine, Calif), an umbrella-like device, designed for percutaneous delivery to the aortic arch, for prevention of cerebral ischemic injury caused by emboli originating in the heart and aorta. Proof-of-concept was shown in a first-in-human study with 4 patients. ${ }^{12}$ We aimed to evaluate the effectiveness of the Embrella device in diminishing the number of post-TAVR cerebral infarcts on DWI, in patients undergoing TAVR with the use of this protection device compared with TAVR procedures without the use of an EPD.

\section{METHODS \\ Patients}

Between September 2012 and July 2013, a total of 58 patients with symptomatic severe aortic stenosis underwent transfemoral TAVR in our institution. During this period, brain magnetic resonance imaging (MRI) had been performed routinely as part of post-TAVR standard care at our center in all patients without contraindications for MRI. From May 2013 onwards, the Embrella Embolic Deflector System was used during TAVR in 15 consecutive patients, without implementing eligibility requirements. These patients were retrospectively compared to the all 37 patients who had undergone TAVR in the previously mentioned period of time, without the use of an EPD and who underwent a postprocedural brain MRI (TAVR-only group). All patients were formally discussed in the heart team and considered inoperable or at high risk for surgical aortic valve replacement because of age, high logistic EuroSCORE, porcelain aorta, malignancy, frailty, or severe comorbidities. As the Embrella device is CE-marked in May 2010, commercially available and used in clinical practice in our series, and as cerebral DWI is part of postprocedural standard care at our center, institutional approval of waiver of informed consent was obtained.

\section{Device and Procedure}

The Embrella Embolic Deflector System is an umbrella-like device that consists of 2 polyurethane membranes mounted on a nitinol frame (Figure 1, A). This device is designed to deflect rather than to capture embolic particles. The polyurethane membrane has $100-\mu \mathrm{m}$ pores to ensure proper blood circulation downstream of the device. The device can be folded, sheathed and loaded into a $6 \mathrm{~F}$ long delivery sheath. It is attached to a 0.035 -inch nitinol delivery cable and can be introduced in the horizontal segment of the aortic arch through either right radial, ulnar, or brachial arteries. Subsequently, the device consisting of 2 petals is released from the sheath, is pulled back, and is positioned at the outer curvature of the aortic arch, such that the petals cover the left carotid and the innominate arteries (Figure 1,B). In some patients, it will further cover (sometimes only partially) the left subclavian artery.

Before beginning the TAVR procedure, apposition of the filter frame in the aortic arch was confirmed with angiography to ensure protection of the cerebral vascular circulation.

Sitting at the outer curvature of the aortic arch, the device does not interfere with the TAVR procedure, and in particular there is no interference with the large valve delivery system (Figure $1, C$ ). Once the procedure is terminated, the device is resheathed using the $6 \mathrm{~F}$ delivery sheath.

TAVR was performed in all cases via the transfemoral approach with the Medtronic CoreValve system (CoreValve Revalving Technology, Medtronic, Minneapolis, Minn) or Edwards SAPIEN XT valve prosthesis (Edwards Lifesciences). Regardless of the prosthesis type, balloon aortic valvuloplasty was performed under rapid pacing to predilate the native aortic valve. The valve prostheses were subsequently deployed under rapid pacing (Edwards: 180 beats/min) or slow pacing (CoreValve: $120-140$ beats/min).

\section{Magnetic Resonance Imaging}

Magnetic resonance imaging was performed within 4 days after TAVR, using a 3-Tesla system (Philips Medical Systems, Best, The Netherlands).

The imaging protocol included a diffusion-weighted single-shot spin echo echoplanar sequence (diffusion gradient $b$ values of 0 and $1000 \mathrm{~s} / \mathrm{mm}^{2}$, repetition time [TR]: 3307 milliseconds, echo time [TE]: 68 milliseconds, 26 slices with a slice thickness of $4 \mathrm{~mm}$, field of view: $230 \mathrm{~mm}$, matrix: $256 \times 205$ ) and a turbo fluid-attenuated inversion recovery (FLAIR; TR/TE $=11,000 / 125$ milliseconds). The acquisition time for the DWI sequences was 69 seconds.

All MRIs were assessed by 2 skilled observers blinded to neurologic status and procedure. Diffuse alterations in the DWI were not regarded as embolic types of lesions. Number of ischemic lesions, overall single-lesion volume, mean lesion volume per patient, total ischemic volume per patient, and location and vascular territories of all focal diffusion abnormalities (bright lesions on DWI) were documented.

\section{Endpoints and Definitions}

The primary endpoint was the number of new ischemic lesions on cerebral DWI. Our secondary endpoints included technical success, defined as successful delivery and retrieval of the Embrella Embolic Defector System, volume and distribution of postprocedural DWI lesions, and clinical outcomes including post-TAVR rates of TIA and stroke and periprocedural cerebrovascular events. According to the VARC-2 criteria, stroke is defined as an acute episode of focal or global neurologic dysfunction caused by the brain, spinal cord, or retinal vascular injury as a result of hemorrhage or infarction. ${ }^{13}$ According to the same criteria, TIA is defined as a transient episode ( $<24$ hours) of focal neurologic dysfunction caused by the brain, spinal cord, or retinal ischemia, without acute infarction. Silent brain 


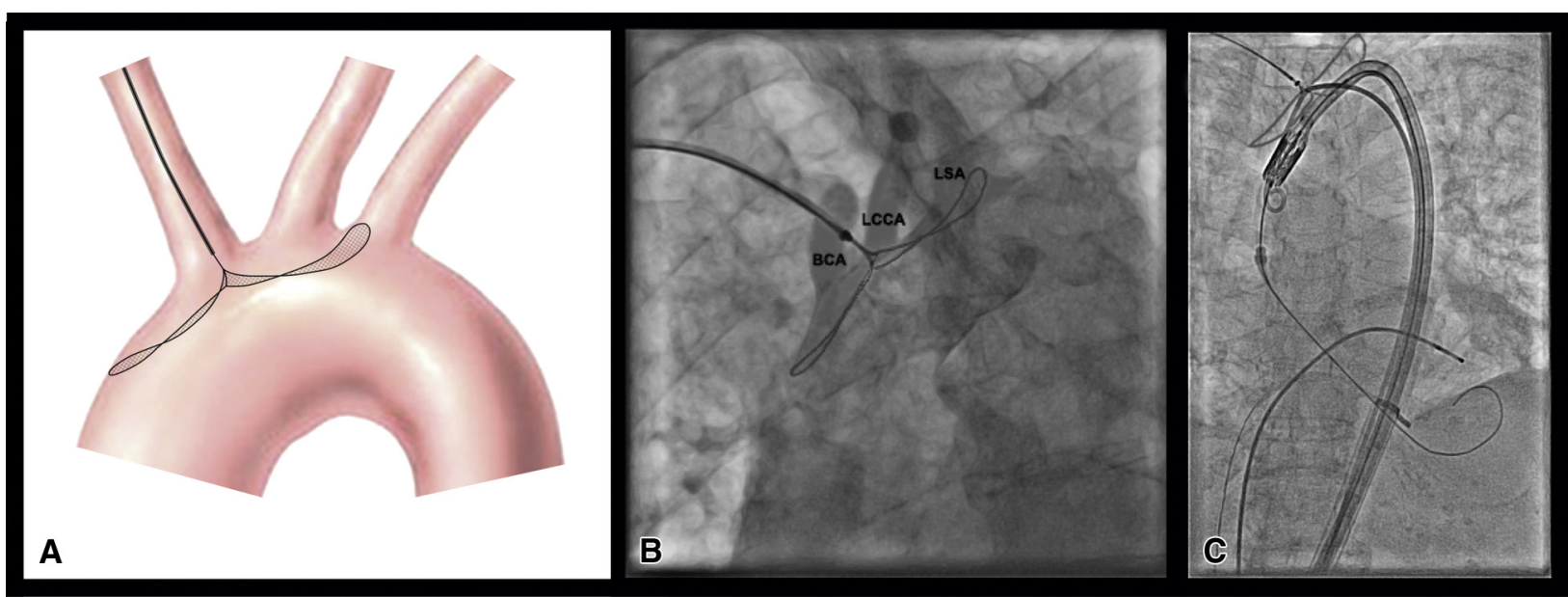

FIGURE 1. A, The Embrella Embolic Deflector System, consisting of 2 petals and a delivery cable. This device is placed at the outer curvature of the aortic arch and the 2 petals cover the brachiocephalic trunk and the left carotid artery. B, Once positioned in the aortic arch, a contrast injection through the delivery sheet confirms proper placement of the device. Note that in this patient, the left subclavian artery also is covered by the Embrella petal. C, Unhampered passage of the valve delivery system through the aortic arch in the presence of the Embrella device. LCCA, Left common carotid artery; LSA, left subclavian artery; $B C A$, brachiocephalic artery.

infarction was defined as new bright lesion on post-TAVR DWI in absence of clinical symptoms.

\section{Statistical Methods}

All analyses were performed with the use of SPSS software (version 20, IBM Corp, Armonk, NY). Continuous variables are expressed as mean \pm standard deviation (SD) or median [interquartile range, IQR]. Categorical variables are described by frequencies and percentages. Comparison of continuous variables was performed using $t$ test or Mann-Whitney $U$ test.
Comparison of categorical variables was performed using Pearson chi-squared test or Fisher exact test and a value of $P<.05$ was considered statistically significant.

\section{RESULTS}

Patient Characteristics and Technical Outcome

Clinical characteristics are presented in Table 1. Technical success was $100 \%$. Correct placement of

TABLE 1. Characteristics of patients and TAVR procedures

\begin{tabular}{|c|c|c|c|}
\hline Characteristics & TAVR alone $(\mathbf{n}=\mathbf{3 7})$ & TAVR + Embrella $(\mathbf{n}=15)$ & $P$ \\
\hline \multicolumn{4}{|l|}{ Patient characteristics } \\
\hline Age (y) & $81[78,84]$ & $84[73,87]$ & .29 \\
\hline Female sex & $16(43)$ & $7(47)$ & .82 \\
\hline Diabetes mellitus & $9(24)$ & $5(33)$ & .51 \\
\hline Hypertension & $21(57)$ & $8(53)$ & .82 \\
\hline Hyperlipidemia & $10(27)$ & $4(27)$ & 1.00 \\
\hline Coronary artery disease & $25(68)$ & $8(53)$ & .33 \\
\hline Previous acute myocardial infarction & $7(19)$ & $1(7)$ & .41 \\
\hline Previous PCI & $20(54)$ & $5(33)$ & .18 \\
\hline Previous CABG & $9(24)$ & $4(27)$ & 1.00 \\
\hline Porcelain aorta according to VARC-2 & $2(5)$ & $1(7)$ & 1.00 \\
\hline Atrial fibrillation & $15(41)$ & $6(40)$ & .97 \\
\hline Carotid disease & $1(3)$ & $2(13)$ & .20 \\
\hline Peripheral vascular disease & $3(8)$ & $1(7)$ & 1.00 \\
\hline Prior stroke or TIA & $5(14)$ & $3(20)$ & .68 \\
\hline Logistic EuroSCORE (\%) & $13.1[8.0,18.1]$ & $16.6[10.7,22.6]$ & .12 \\
\hline \multicolumn{4}{|l|}{ Procedural characteristics } \\
\hline Fluoroscopy time (min) & $17.8[12.9,20.0]$ & $22.0[18.8,26.0]$ & .005 \\
\hline Contrast medium (mL) & $145.0[125.3,180.0]$ & $160.0[150.0,190.0]$ & .12 \\
\hline \multicolumn{4}{|l|}{ TAVR prosthesis type } \\
\hline Edward SAPIEN XT & $22(59)$ & $11(73)$ & .35 \\
\hline Medtronic CoreValve & $15(41)$ & $4(27)$ & .35 \\
\hline
\end{tabular}

Data are shown as median [interquartile range] or $\mathrm{n}(\%) . T A V R$, Transcatheter aortic valve replacement; $P C I$, percutaneous coronary intervention; $C A B G$, coronary artery bypass grafting; TIA, transient ischemic attack; VARC-2, Valve Academic Research Consortium-2; EuroSCORE, European System for Cardiac Operative Risk Evaluation. 
the Embrella device with the deflecting petals covering the brachiocephalic and left carotid artery ostia was achieved in all but 1 patient. One patient showed anomaly of the aortic arch, with the right carotid and an aberrant right subclavian artery (arteria lusoria) arising directly from the aorta (Figure E1). In this patient, left and right carotid and left subclavian arteries were not covered by the Embrella device. All other patients had type II aortic arch anatomy, with the right subclavian and right carotid artery originating from the brachiocephalic artery. The access site was radial in $13(87 \%)$ and ulnar in $2(13 \%)$ patients. In 6 cases $(40 \%)$, the ostium of the left subclavian artery also was covered.

With regard to the implantation procedure of the aortic valve prosthesis, advancement of the transfemoral valve delivery system alongside the Embrella device was associated with minimal interaction without significant interference or dislodgment of the device. Device success as defined by VARC-2 definitions was achieved in all patients in the Embrella group and 35 patients $(95 \%)$ in the TAVR-only group $(P=1.00)$. Reasons for not fulfilling the device success criteria were additional valve implantation in one patient in the TAVR-only group due to too low placement of the Medtronic CoreValve prosthesis and hence severe paravalvular regurgitation, and residual moderate paravalvular regurgitation after (Edwards SAPIEN XT) valve implantation in a second patient in the same group.

After valve implantation and eventual additional attempts to optimize the result, the Embrella device was withdrawn by "collapsing" the 2 nitinol petals into the delivery sheath. Hemostasis of the radial or ulnar access site was accomplished with a standard compression band. No vascular and bleeding complications occurred. The total fluoroscopy time was significantly longer in the Embrella group compared with the TAVR-only group $(23.2 \pm 7.0$ vs $18.0 \pm 6.9$, Median: $22.0[18.8,26.0]$ vs 17.8 [12.9, 20.0], $P=.005$ ) (Table 1).

\section{Primary, DWI Outcome}

As summarized in Table 2, post-TAVR DWI revealed new ischemic lesions in all patients in the Embrella group and in $35(95 \%)$ cases in the TAVR-only group. Lesions were typically multiple in both groups with a significantly higher number of lesions in the Embrella group: a median of 9.0 lesions [4.0 to 12.0] in the Embrella group and 5.0 lesions [2.0 to 7.0$]$ in the TAVR-only group $(P=.044)$. The use of the Embrella device was, however, associated with a significant reduction in single-lesion volume: $9.7 \mu \mathrm{L}[5.8,18.4]$ versus $17.8 \mu \mathrm{L}[9.5,38.7](P<.001)$. Ischemic lesions in the Embrella group were more frequently of the smallest size $(<20 \mu \mathrm{L})$ compared to the TAVR-only group $(80 \%$ vs $54 \%, P<.001)$ (Figure $2, A)$. Concerning the total volume of new ischemic lesions (total infarct volume), no significant difference was observed between the 2 groups: $111.6 \mu \mathrm{L}$ [65.0 to 184.1] in the Embrella group and $129.4 \mu \mathrm{L}$ [53.0 to 357.0] in the TAVR-only group $(P=.98)$. However, total infarction volumes of more than $1000 \mu \mathrm{L}$ occurred only in the TAVR-only group (in $8.1 \%$ of cases) (Figure 2, B).

New ischemic lesions were located in the cerebellum and cortex, deep white matter, and deep gray matter in the affected cerebral hemisphere. A higher frequency of cortical lesions in the perfusion territory of anterior cerebral artery was detected in the Embrella group compared with the TAVR-only group (28[18\%] vs $24[9 \%], P=.02$; Table 3). No other differences were observed in the distribution pattern of DWI lesions between the 2 groups. Whereas lesions were detected almost equally in left and right cerebral and cerebellar hemispheres in the TAVR-only group, more lesions were detected in the right side of the brain in the Embrella group (Figure 2, C).

\section{Secondary, Clinical Outcome}

No procedural complications occurred; in particular, there were no complications related to insertion of the Embrella device. During in-hospital follow-up, no patient developed new neurologic symptoms or clinical findings

TABLE 2. Findings on DWI after TAVR

\begin{tabular}{lccc}
\hline \multicolumn{1}{c}{ Finding } & TAVR alone $(\mathbf{n}=\mathbf{3 7})$ & TAVR + Embrella $(\mathbf{n}=\mathbf{1 5})$ & \multicolumn{1}{c}{$\boldsymbol{P}$} \\
\hline Patient with new lesions & $35(95)$ & $15(100)$ & 1.00 \\
Patient with single lesion & $4(11)$ & $0(0)$ & .31 \\
Patients with multiple lesions & $31(84)$ & $15(100)$ & .17 \\
Lesions per patient & $5.0[2.0,7.0]$ & $9.0[4.0,12.0]$ & .044 \\
Right hemisphere and cerebellum & $2.0[0.5,4.0]$ & $5.0[2.0,10.0]$ & .008 \\
Left hemisphere and cerebellum & $2.0[1.0,5.0]$ & $3.0[2.0,6.0]$ & .34 \\
Lesion volume $(\mu \mathrm{L})$ & & $15.2[10.9,21.7]$ \\
Mean per patient & $25.1[11.0,61.0]$ & $111.6[65.0,184.1]$ & .092 \\
Total ischemic volume per patient & $129.4[53.0,357.0]$ & $68.4[20.6,126.8]$ & .98 \\
Total ischemic volume: right side & $60.3[1.2,132.4]$ & $51.3[32.1,83.8]$ & .63 \\
Total ischemic volume: left side & $65.3[10.4,191.3]$ & .80 \\
\hline
\end{tabular}

Data are shown as median [interquartile range] or $\mathrm{n}(\%) . T A V R$, Transcatheter aortic valve replacement. 

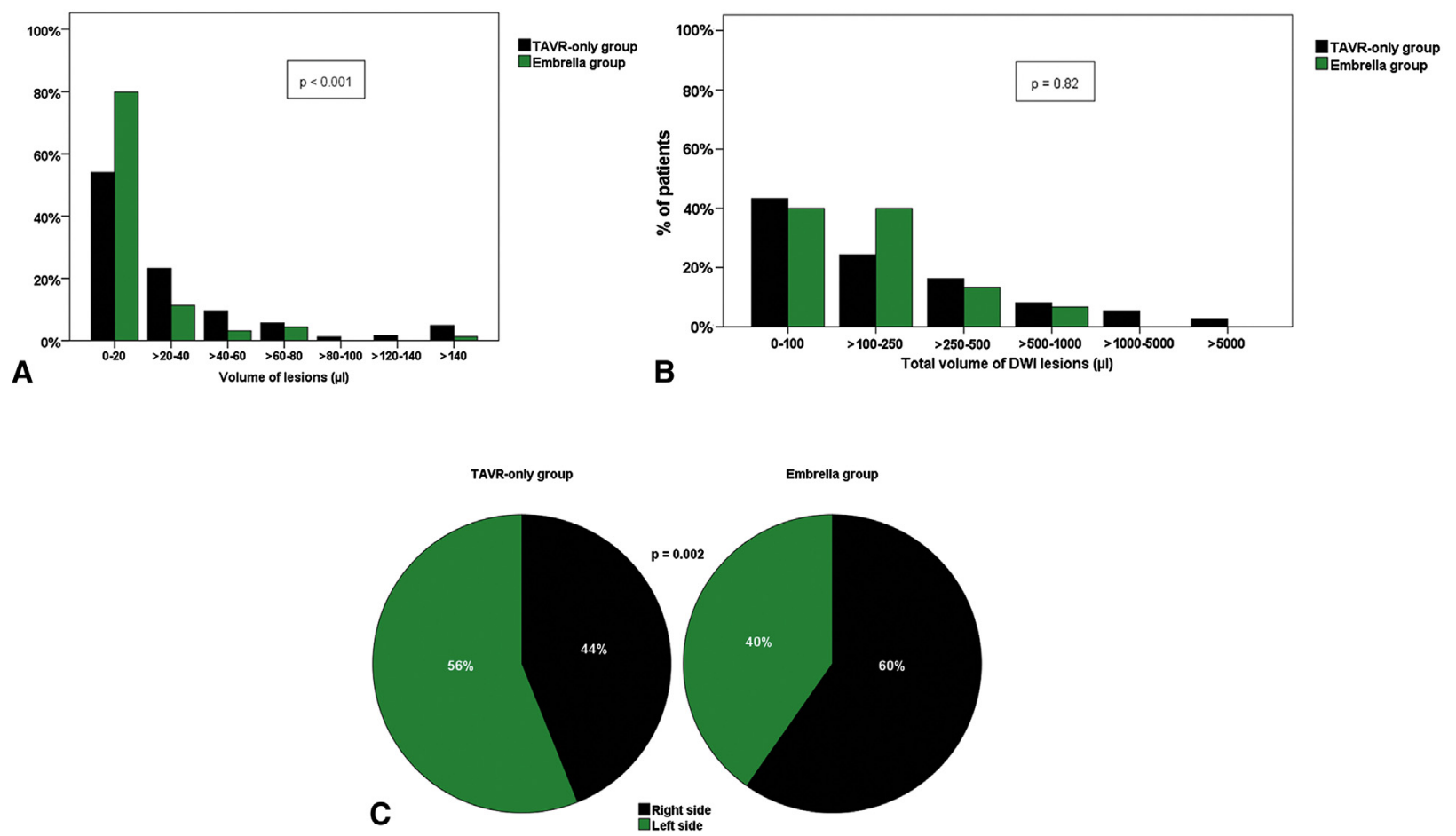

FIGURE 2. A, Incidence of different sizes of cerebral DWI lesions in the Embrella group compared to the TAVR-only group. DWI lesions were categorized according to volume. The majority of lesions in the Embrella group have a volume of less than $20 \mu \mathrm{L}(80 \%)$, which is the case in only $54 \%$ of the TAVR-only group. B, Lesion volumes of more than $1000 \mu \mathrm{L}$ occurred only in the TAVR-only group. C, Left/right distribution of DWI lesions. A shift toward a higher number of lesions in the right side of the brain was observed when an Embrella Embolic Deflector System was used during TAVR. DWI, Diffusion weighted magnetic resonance imaging; TAVR, transcatheter aortic valve replacement.

of stroke except for 1 patient in the TAVR-only group who suffered from dysphasia, diagnosed as TIA, 30 minutes after the procedure. MRI performed after TAVR confirmed a new ischemic lesion $(40 \mu \mathrm{L})$ in the site corresponding to the symptoms in this patient (Figure E2). No patient suffered from clinically relevant peripheral embolization toward nonbrain regions.

\section{DISCUSSION}

Minimizing the risk of procedure-related cerebral injury is of paramount importance to the overall success of TAVR, especially if this procedure has the potential to become a treatment option also for younger patients with severe aortic stenosis. The passage of bulky devices through a diseased aorta and the aggressive manipulation of a stenotic,

TABLE 3. DWI lesion analysis

\begin{tabular}{lccc}
\hline \multicolumn{1}{c}{ Lesion } & TAVR alone $(\mathbf{n}=\mathbf{2 5 4})$ & TAVR + Embrella $(\mathbf{n}=\mathbf{1 5 9})$ & $\boldsymbol{P}$ \\
\hline Cortical infarcts & $123(48)$ & $86(54)$ & .26 \\
Anterior cerebral artery & $24(9)$ & $28(18)$ & .02 \\
Middle cerebral artery & $47(19)$ & $27(17)$ & .70 \\
Posterior cerebral artery & $22(9)$ & $9(6)$ & .26 \\
Border zone & $30(12)$ & $22(14)$ & .55 \\
Subcortical infarcts & $75(30)$ & $37(23)$ & .16 \\
Internal border zone & $12(5)$ & $13(8)$ & .13 \\
Deep gray matter & $11(4)$ & $23(14)$ & .10 \\
Infratentorial & $45(18)$ & $21(13)$ & .39 \\
Cerebellum & $41(16)$ & $2(1)$ & .42 \\
Brainstem & $4(2)$ & $9.7[5.8,18.4]$ & \\
Single-lesion volume $(\mu \mathrm{L})$ & $17.8[9.5,38.7]$ & $9.5[5.1,19.3]$ & $<.00$ \\
Right side & $17.6[9.2,35.0]$ & $10.4[7.3,15.8]$ & $<.001$ \\
Left side & $18.6[9.8,39.6]$ &
\end{tabular}

Data are shown as median [interquartile range] or $\mathrm{n}(\%) . T A V R$, Transcatheter aortic valve replacement. 
calcified aortic valve could cause embolization of atherosclerotic material, clots, and other calcified debris. The need to reduce TAVR-related cerebral complications has spurned significant research and the development of EPDs. The use of an EPD during TAVR is not a novel idea, and benefits of these types of devices for brain protection have been demonstrated in carotid intervention. ${ }^{11} \mathrm{~A}$ systematic review including 896 carotid stenting cases with EPDs and 2537 cases without EPDs found that EPD usage was associated with significantly lower stroke rates $(1.8 \%$ vs $5.5 \%, P<.001) .{ }^{14}$

In the present study, we investigated the benefit of the use of the Embrella Embolic Deflector System for brain protection during TAVR procedures. Initial animal work demonstrated that Embrella was effective in deflecting emboli ranging in size from 150 to $600 \mu \mathrm{m}$ away from the carotid circulation. ${ }^{15}$ Retrieved particulate matter was reduced in the carotid filtration circuit from 19\% (during unprotected aortic injection of prepared human atheroma) to $1.3 \%$ with Embrella usage $(P<.001)$. Nietlispach et $\mathrm{al}^{12}$ have described their initial in-human experience with the Embrella device in 3 transfemoral TAVR patients (with the Edwards Sapien valve) and 1 balloon aortic valvuloplasty case. No clinically overt stroke was found, but postprocedure DWI revealed a new ischemic lesion in the balloon valvuloplasty patient. DWI data in our study revealed a significantly higher number of lesions in the Embrella group compared with the TAVR-only group. An increase in the number of ischemic lesions may be caused by the introduction and recapture of the Embrella device, manipulations that may inadvertently lead to cerebral showering caused by dislodgment of atherocalcific material from the brachiocephalic artery and aortic arch. Dislodgment of atherocalcific material in the brachiocephalic artery may cause direct cerebral embolization. The latter would theoretically cause an increased number of embolic DWI lesions in the right side of the brain. Our data support the aforementioned hypothesis as we showed a significant increase in the number of DWI lesions in the right half of the brain in the Embrella group compared with the TAVR-only group. Also, a trend toward higher total infarct volumes was found in the right side of the brain in the Embrella group. This phenomenon was not observed in the TAVR-only group (Table 2). These observations suggest a possible increase in the ischemic burden for the right side of the brain due to right radial/brachial access. Considering that the elderly TAVR patient candidates are at a high risk of having thrombogenic plaques in the brachiocephalic artery and aortic arch, transfemoral introduction of the EPD might be a safer approach for the brain.

Interestingly, the present study showed a significant reduction in lesion volume on DWI and a trend toward lower total infarct volume because of the use of an Embrella device and especially the absence of large-volume lesions. The latter observation may be explained by the deflection of large embolic particles, originating from the calcified aortic valve, by the Embrella device during TAVR. Moreover, small emboli may arise distal to the Embrella device during its introduction and recapture. This could explain the larger number of smaller lesions in the Embrella group. Reduction in total infarct volumes in the Embrella group may decrease the risk of future neurocognitive impairment, though not supported by the available evidence, and it needs further investigation.

Almost all studies to date investigating the benefit of the EPDs for brain protection during TAVR have reported postprocedural occurrence of new DWI lesions, even with the use of an EPD (Table E1). ${ }^{12,16-18}$ This (partial) lack in full effectiveness could be explained by different factors. First of all, lack of coverage of the left vertebral arterial system by the Embrella device and the Claret Medical diminishes the usefulness of these types of EPDs. Second, thromboembolic events that cause cerebral injury are not merely limited to the actual TAVR procedure. Interestingly, as stated previously by other reports, the risk of stroke, though highest in the intraprocedural period, persists following successful TAVR. ${ }^{1,3}$ Therefore, in addition to the use of an effective EPD, there also may be an important role for optimal medication regimen after TAVR in order to decrease the risk of postprocedural cerebrovascular events. The optimal post-TAVR drug therapy to achieve this aim remains unclear, however. Most physicians routinely continue dual antiplatelet therapy (aspirin and clopidogrel) for a number of months. Although not indicated because of lack of evidence, there may be a role for systemic anticoagulation in a role similar to surgical aortic valve replacement with bioprosthethic valves. However, a large number of TAVR patients, given their comorbidities, are likely to be at an increased risk of hemorrhagic complications. Future studies are necessary to address these issues.

In addition to the search for an efficient cerebral protection during and after TAVR, more attention needs to be paid to preprocedural patient evaluation and assessment. A thorough evaluation of risk factors for cerebrovascular events with multimodality imaging may aid risk stratification and guide the approach for valve delivery. For instance, significant atheroma in the aortic arch may favor a transapical approach, although this has not been proved by DWI or clinical reports to date. Nonetheless, further refinements to EPD technique may offer a modality for significant reduction of brain injury during TAVR. Future studies need to elucidate the benefit of improved EPD technology for use during TAVR on the one hand and the impact of usually silent DWI lesions on neurocognitive function of patients undergoing these procedures on the other hand. 


\section{Limitations}

This study has a number of limitations. First, because of the nonrandomized trial design, a selection bias cannot be excluded. Second, the number of patients treated with the Embrella device is small and therefore a definitive conclusion regarding the effectiveness of this device cannot be made. Finally, our study lacks formal neurocognitive assessment before and after the procedures in order to capture subtle changes in the neurologic and cognitive status of patients. A few small studies have recently focused on the benefit of EPD usage during TAVR procedures, although the real clinical impact of asymptomatic DWI lesions is still debatable. Future studies are needed to address this issue more thoroughly.

\section{CONCLUSIONS}

The current study shows that the use of the Embrella Embolic Deflector System during TAVR procedures is associated with a higher number of postprocedural cerebral DWI lesions compared with procedures without EPD usage. This increase in number is however accompanied by a significant reduction in single-lesion volume and the absence of large total infarct volumes $(>1000 \mu \mathrm{L})$, reflecting the potential of the Embrella device to prevent large-volume ischemic lesions. The reduction in single-lesion volume may reduce cerebral ischemic burden during TAVR and needs further investigation in future studies with a larger number of patients.

\section{Conflict of Interest Statement}

Pieter R. Stella is a physician proctor for Edwards Lifesciences. All other authors have nothing to disclose with regard to commercial support.

\section{References}

1. Rodes-Cabau J, Webb JG, Cheung A, Ye J, Dumont E, Feindel CM, et al. Transcatheter aortic valve implantation for the treatment of severe symptomatic aortic stenosis in patients at very high or prohibitive surgical risk: acute and late outcomes of the multicenter Canadian experience. J Am Coll Cardiol. 2010;55:1080-90.

2. Gilard M, Eltchaninoff H, Iung B, Donzeau-Gouge P, Chevreul K, Fajadet J, et al. Registry of transcatheter aortic-valve implantation in high-risk patients. $N$ Engl J Med. 2012;366:1705-15.

3. Leon MB, Smith CR, Mack M, Miller DC, Moses JW, Svensson LG, et al. Transcatheter aortic-valve implantation for aortic stenosis in patients who cannot undergo surgery. N Engl J Med. 2010;363:1597-607.
4. Arnold M, Schulz-Heise S, Achenbach S, Ott S, Dorfler A, Ropers D, et al. Embolic cerebral insults after transapical aortic valve implantation detected by magnetic resonance imaging. JACC Cardiovasc Interv. 2010;3:1126-32.

5. Ghanem A, Muller A, Nahle CP, Kocurek J, Werner N, Hammerstingl C, et al. Risk and fate of cerebral embolism after transfemoral aortic valve implantation: a prospective pilot study with diffusion-weighted magnetic resonance imaging. $J$ Am Coll Cardiol. 2010;55:1427-32.

6. Rodes-Cabau J, Dumont E, Boone RH, Larose E, Bagur R, Gurvitch R, et al. Cerebral embolism following transcatheter aortic valve implantation: comparison of transfemoral and transapical approaches. J Am Coll Cardiol. 2011;57:18-28.

7. Fairbairn TA, Mather AN, Bijsterveld P, Worthy G, Currie S, Goddard AJ, et al. Diffusion-weighted MRI determined cerebral embolic infarction following transcatheter aortic valve implantation: assessment of predictive risk factors and the relationship to subsequent health status. Heart. 2012;98:18-23.

8. Kahlert P, Knipp SC, Schlamann M, Thielmann M, Al-Rashid F, Weber M, et al Silent and apparent cerebral ischemia after percutaneous transfemoral aortic valve implantation: a diffusion-weighted magnetic resonance imaging study Circulation. 2010;121:870-8

9. Knecht S, Oelschlager C, Duning T, Lohmann H, Albers J, Stehling C, et al Atrial fibrillation in stroke-free patients is associated with memory impairment and hippocampal atrophy. Eur Heart J. 2008;29:2125-32.

10. Vermeer SE, Prins ND, den Heijer T, Hofman A, Koudstaal PJ, Breteler MM. Silent brain infarcts and the risk of dementia and cognitive decline. N Engl J Med. 2003;348:1215-22.

11. Atkins MD, Bush RL. Embolic protection devices for carotid artery stenting: have they made a significant difference in outcomes? Semin Vasc Surg. 2007; 20:244-51.

12. Nietlispach F, Wijesinghe N, Gurvitch R, Tay E, Carpenter JP, Burns C, et al. An embolic deflection device for aortic valve interventions. JACC Cardiovasc Interv. 2010;3:1133-8.

13. Kappetein AP, Head SJ, Genereux P, Piazza N, van Mieghem NM Blackstone EH, et al. Updated standardized endpoint definitions for transcatheter aortic valve implantation: The Valve Academic Research Consortium-2 consensus document. J Thorac Cardiovasc Surg. 2013;145:6-23.

14. Kastrup A, Groschel K, Krapf H, Brehm BR, Dichgans J, Schulz JB. Early outcome of carotid angioplasty and stenting with and without cerebral protection devices: a systematic review of the literature. Stroke. 2003;34:813-9.

15. Carpenter JP, Carpenter JT, Tellez A, Webb JG, Yi GH, Granada JF. A percutaneous aortic device for cerebral embolic protection during cardiovascular intervention. J Vasc Surg. 2011;54:174-81.e1.

16. Etienne PY, Papadatos S, Pieters D, El Khoury E, Alexis F, Price J, et al. Em bol-X intraaortic filter and transaortic approach for improved cerebral protection in transcatheter aortic valve implantation. Ann Thorac Surg. 2011;92: e95-6.

17. Onsea K, Agostoni P, Samim M, Voskuil M, Kluin J, Budde R, et al. First-in-man experience with a new embolic deflection device in transcatheter aortic valve interventions. EuroIntervention. 2012;8:51-6.

18. Naber CK, Ghanem A, Abizaid AA, Wolf A, Sinning JM, Werner N, et al. Firstin-man use of a novel EPD for patients undergoing transcatheter aortic valve implantation. EuroIntervention. 2012;8:43-50.

Key words: aortic valve stenosis, transcatheter aortic valve replacement, cerebral ischemic injury, intracranial embolism, cerebral embolic protection device, Embrella Embolic Deflector System 


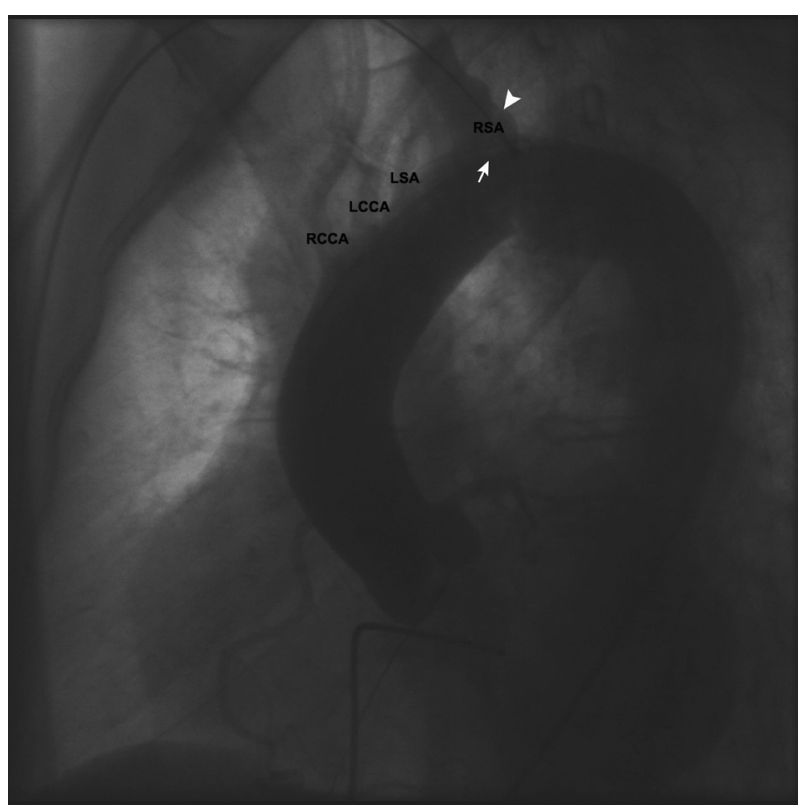

FIGURE E1. Rotational angiography image showing Embrella device (arrow) placed in the aortic arch of a patient with an arteria lusoria: anomaly of the aortic arch, with the right carotid and an aberrant right subclavian artery arising directly from the aorta. The arteria lusoria (arrow head) arises beyond the left subclavian artery. In this patient, the left and right carotid and left subclavian arteries were not covered by the Embrella device. $L C C A$, Left common carotid artery; $L S A$, left subclavian artery; RCCA, right common carotid artery; $R S A$, right subclavian artery.

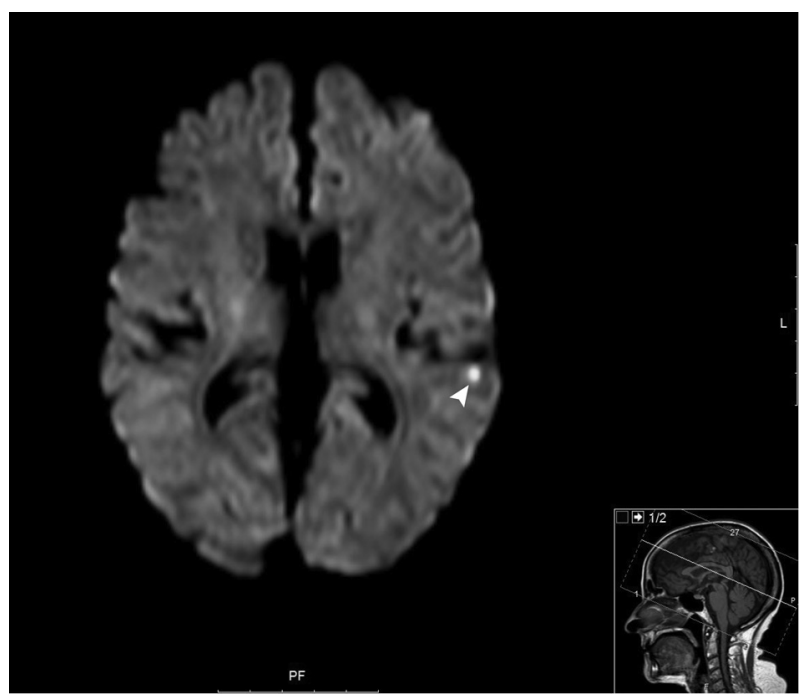

FIGURE E2. DWI showing a TIA-related lesion of $40 \mu \mathrm{L}$ in the Wernick area (arrow head). The patient suffered from transient signs of dysphasia. 
TABLE E1. Embolic protection devices studied for usage during TAVR

\begin{tabular}{|c|c|c|c|c|c|c|c|c|}
\hline Device & Access site & $\begin{array}{c}\text { Mechanism of } \\
\text { work }\end{array}$ & $\begin{array}{c}\text { Pore size } \\
(\mu \mathrm{m})\end{array}$ & Study & $\begin{array}{l}\text { No. of cases in } \\
\text { the study }\end{array}$ & TIA/Stroke & $\begin{array}{c}\% \text { Patients } \\
\text { with DWI } \\
\text { lesions }\end{array}$ & $\begin{array}{c}\text { Average DWI } \\
\text { lesions per patien }\end{array}$ \\
\hline \multirow[t]{2}{*}{ Embrella } & \multirow{2}{*}{$\begin{array}{c}\text { Radial/ulnar/ } \\
\text { brachial }\end{array}$} & \multirow[t]{2}{*}{ Deflecting } & \multirow[t]{2}{*}{100} & Nietlispach et $\mathrm{al}^{12}$ & 4 & $0 \%$ & 25 & 0.25 \\
\hline & & & & Samim et al & 15 & $0 \%$ & 100 & 10.8 \\
\hline SMT & Femoral & Deflecting & $\sim 200$ & Onsea et $\mathrm{al}^{17}$ & 15 & - & 100 & 3.2 \\
\hline Claret Medical & Radial/brachial & Capturing & 140 & Naber et $\mathrm{al}^{18}$ & 35 & $0 \%$ & - & - \\
\hline Embol-X & Transaortic & Capturing & 120 & Etienne et $\mathrm{al}^{16}$ & 1 & $0 \%$ & 0 & 0 \\
\hline
\end{tabular}

TIA, Transient ischemic attack; DWI, diffusion weighted magnetic resonance imaging; SMT, SMT Embolic Deflection Device (SMT Research and Development Ltd, Herzliya, Israel). 